\title{
REMARKS UPON PROFESSOR RÜCKER'S PAPER AND WILDE'S MAGNETARIUM.
}

By L. A. BaUER.

I am constrained to add a few remarks upon the foregoing paper and the interpretation to be put upon the results derived from the "Magnetarium." Professor Rücker admits that Wilde's results must be received with great caution, and that the conditions under which the experiments were performed are open to legitinate criticism. As I have already had occasion in another connection to point out some of the errors to which "the Magnetarium" results are liable, I shall not enter again upon this matter, but simply accept Wilde's observational results as correct, and endeavor to show what interpretation is to be put upon them.

As Professor Rücker states, "The magnetic field of the earth may be regarded as that primarily due to a uniformly magnetized globe disturbed by some secondary causes." Furthermore, the result of recent investigations has been that this field originates practically from causes within the earth's crust, and can be referred to a potential. The total magnetic potential on the earth's surface can then be represented by the following terms:

$$
V=V_{s}+V_{s}^{\prime}+V_{u} ; V_{s}=V_{p}+V_{e} ; V_{s}^{\prime}=V_{p}^{\prime}+V_{e}^{\prime} .
$$

$V_{s}+V^{\prime}{ }_{s}$ together represent all that portion which can be referred to a uniform magnetization about some diameter inclined to the earth's rotation axis, $V^{\prime} s$ being that portion which can be regarded as induced by $V_{s}$. We can resolve $V_{s}$ and $V^{\prime}$ seach into two component parts, one symmetrical about the rotation axis, $V_{p}$ and $V_{p}^{\prime}$, and the other symmetrical about diameters in the equatorial plane, viz., $V_{e}$ and $V_{e}^{\prime}$, respectively. The ratio of $\left(V_{p}+V_{p}^{\prime}\right)$ to $\left\langle V_{c}+V_{e}^{\prime}\right\rangle$ is nearly as 5 to $\mathrm{I}$.

The problem now is to determine $V_{u}$, the unsymmetrical portion of the earth's magnetism; i. e., that portion which can not be referred to a simple magnetization about a diameter of the earth, and to ascertain the causes producing the unsymmetrical magnetization. The general assumption amounts to this: the earth has a symmetrical primary field; secondly, a symmetrical secondary field; and, thirdly, an unsymmetrical secondary field. It is then very evident that the accurate determination of $V_{u}$ depends upon the accurate elimination of the symmetrical portion, $V_{s}^{\prime}+V^{\prime}$.

Now, in the present state of our knowledge, we can build up the symmetrical field in an infinite number of ways. Wilde has done this in one way. He starts with a primary resultant field, approximately, tuned to one station, London. He finds that this field does not yet represent the general magnetic phenomena. After 
various trials, he covers the ocean areas (three-fourths of the earth's surface) with sheet-iron, adds polarizing bands over the land areas here and there until he has obtained some of the striking nuagnetic phenomena. He then concludes that the primary cause of the unsymmetrical distribution of the earth's magnetism is to be referred to the fact assumed; viz., that the earth beneath the oceans is more highly magnetizable than that beneath the continents.

When Wilde covers almost the entire earth (over three-fourths) with sheet-iron, he has added practically a third field to his two primary ones, the effects from which would consist largely of another symmetrical magnetization. In other words, before covering the ocean areas with sheet-iron and adding the various polarizing bands over land areas, he had not yet succeeded in obtaining even the phenonena of the symmetrical portion of the earth's magnetism. The amount and disposition of the sheet-iron pieces depend entirely upon the primary fields we start with. Having no means at present for deriving the precise primary field, we may start in an infinite number of ways and get an infinite number of arbitrary results. It is, therefore, apparent that we can not as yet accept the validity of Wilde's conclusion as to the cause of the unsymmetrical distribution of the earth's magnetism.

Professor Rücker has pointed out, by an example, that it is not sufficient to reproduce only certain ones of the observed magnetic phenomena. I have no hesitation in asserting that, if an independent, unbrased investigation is made of the results of the Wilde Magnetarizm for the various elements of the earth's magnetism, certainly just as many, if not more, discordances as accordances will be found between the actually observed results and the Magnetarium results. The same conclusion I reached some time ago with regard to his secular variation results.

Reference has been made in the previous article to my residual field as illustrated in the paper in the March issue of the JOURNAL. My published map is based, not only upon certain observed features of the earth's magnetism over certain regions, but upon all the observed elements at $I, 800$ points symmetrically distributed over the earth's surface between latitudes $60^{\circ} \mathrm{N}$. and $60^{\circ} \mathrm{S}$. All that portion (whether due to inducing or to induced fields) which could be referred to a uniform magnetization about a diameter inclined to the earth's axis was first eliminated. I accepted for this purpose, Schnidt's results as derived from his elaborate investigations. The first-order terms of the Gaussian expansion will not necessarily give the maximum amount of the earth's magnetism that may be due to such a simple magnetization, and so, some years ago, I made a preliminary investigation towards ascertaining how much the result from the Gaussian expansion would differ from the maximum result, and 
found it to be very small. I shall enter upon this matter more fully in my next paper, and likewise deduce some interesting facts pertaining to the symmetrical field. The only point I desire to make here is, that my residual field is not that which Wilde gets when he adds the sheet-iron coverings and bands; for, as shown above, in his field there lurks still a large part of symmetrical magnetization.

My residual field does not reveal the fact that the cause of the unsymmetrical distribution is due to the earth under the ocean areas being more magnetic than below the continental areas. It does show, however, apparently, that the primary cause of the unsymmetrical distribution is connected in some manner with the distribution of land and water; for, after a careful comparison made by myself and others, the final conclusion reached was, that the "unsymmetrical distribution of the earth's magnetism and the unsymmetrical distribution of temperature as exhibited on the earth's surface, on the average for the year, are in some way related to each other." I was careful not to say that one was the cause of the other, although some facts revealed might have warranted such a conclusion. I prefer to leave the assigning of the cause open as yet; for both phenomena, the unsymmetrical temperature distribution and the unsymmetrical magnetic distribution, may not necessarily be connected with each other as cause and effect, but may both be effects from the same cause; viz., the distribution of land and water, ocean currents, etc. Hence, the guarded way in which I formulated my conclusion. That there certainly is a remarkable similarity between the map of the isabnormal temperatures and that of the residual magnetization, a careful inspection will show.

Professor Rïcker points out an objection to the possibility of such a connection; viz., that the "deviation of the average temperature of any points at the surface of the ocean from the average for the corresponding latitude is a surface plenomenon, which, at points near the bottom of the ocean, is completely masked by the influx of cold water." The annual isabnormal temperatures may be regarded as referring to a layer at a depth beyond the influence of the diurnal and the annual temperature variations, say about 50 to 60 feet on the average. Professor Rïcker forgets that the magnetic elements which we are compelled to deal with are also surface phenomena. We know absolutely nothing of the actual distribution of the magnetic elements at the bottom of the oceans. Here is a wonderful opportunity for scientific investigation! However, that great temperature differences are bound to exist along parallels of latitude at the average depth of the oceans is a well-known fact. It does not seem to me, therefore, that Professor Rücker's objection is as yet a valid one. 